\title{
A Comparison of the Body Composition Changes Between Laparoscopy-assisted and Open Total Gastrectomy for Gastric Cancer
}

\author{
TORU AOYAMA $^{1,2^{*}}$, TAKAKI YOSHIKAWA ${ }^{1,2^{*}}$, YUKIO MAEZAWA ${ }^{1}$, KAZUKI KANO $^{1}$, KENTARO HARA $^{1}$, \\ TSUTOMU SATO $^{1}$, TSUTOMU HAYASHI $^{2}$, TAKANOBU YAMADA ${ }^{2}$, HARUHIKO CHO $^{1}$, TAKASHI OGATA ${ }^{1}$, \\ HIROSHI TAMAGAWA ${ }^{2}$, NORIO YUKAWA ${ }^{2}$, YASUSHI RINO ${ }^{2}$, MUNETAKA MASUDA $^{2}$ and TAKASHI OSHIMA ${ }^{1,2}$ \\ ${ }^{1}$ Department of Gastrointestinal Surgery, Kanagawa Cancer Center, Kanagawa, Japan; \\ ${ }^{2}$ Department of Surgery, Yokohama City University, Yokohama, Japan
}

\begin{abstract}
Background/Aim: Laparoscopy-assisted total gastrectomy (LATG) for gastric cancer may prevent the loss of body weight or lean body mass after surgery due to its reduced surgical stress compared with open total gastrectomy (OTG). Patients and Methods: A total of 303 patients were examined in this study. All patients received the same perioperative care via fast-track surgery. The body weight and composition were evaluated using a bioelectrical impedance analyzer within 1 week before and at 1 week, 1 month, and 3 months after surgery. Results: Two hundred and eight patients received OTG, and 95 received LATG. Although the clinical $T$ factor and $N$ factor were significantly different between these two groups, other clinical factors were similar. The respective body weight loss (1 week/ 1 month/3 months) was $-4.7 \% /-8.0 \% /-11.9 \%$ in the OTG group and $-4.7 \% /-8.2 \% /-11.6 \%$ in the LATG group, that were not significantly different between the two groups at any time point of measurement ( $p=0.698 / 0.528 / 0.534$, respectively). The respective lean body mass loss (1 week/1 month/3 months) was $-4.2 \% /-6.4 \% /-7.4 \%$ in the OTG group and -4.0\%/-5.8\%/-6.2\% in the LATG group, that were not significantly different between the groups ( $p=0.503 / 0.588 / 0.946$, respectively). Conclusion: The body composition changes were similar between the OTG and LATG groups using the same
\end{abstract}

This article is freely accessible online.

*These Authors contributed equally to this study.

Correspondence to: Toru Aoyama, Department of Surgery, Yokohama City University, 3-9 Fukuura, Kanazawa-ku, Yokohama 236-0004, Japan. Tel: +81 453915761, Fax: +81 453614692, e-mail: t-aoyama@lilac.plala.or.jp

Key Words: Body composition, lean body mass, laparoscopyassisted total gastrectomy, open total gastrectomy, surgical stress. perioperative care of fast-track surgery. Adopting a laparoscopic approach would not help in reducing loss of body weight or lean body mass after gastric cancer surgery.

Gastric cancer (GC) is the fourth-most common malignant disease and the second-most frequent cause of cancer-related deaths worldwide (1). Complete resection is essential for the cure of localized gastric cancer $(2,3)$. The use of laparoscopy-assisted total gastrectomy (LATG) to treat gastric cancer was first described by Kitao in 1994 (4). Since then, the number of cases of gastric cancer treated with laparoscopy-assisted total gastrectomy (LATG) has also been gradually increasing. The advantages of this procedure compared with open total gastrectomy (OTG) include reduced amounts of operative blood loss and pain, earlier recovery of bowel activity and the resumption of oral intake and shorter hospital stays (5). Some reports have also suggested lower levels of surgical stress and a lower incidence of impaired nutrition with the laparoscopic approach than with the conventional approach (6).

Recently, we found that the changes in the body weight and body composition after gastrectomy affect the continuation of adjuvant chemotherapy and the patient's survival (7-9). Therefore, minimizing the body weight loss and body composition changes after gastrectomy is needed. Theoretically, less-invasive surgical procedures inhibit muscle catabolism during surgery. Once surgical stress has occurred, immune cells produce cytokines that act as mediators of both immune and systemic responses to injury (10). These cytokines are synthesized from amino acids supplied by muscle catabolism. In addition, the response of skeletal muscle during critical illness is characterized by a rapid decrease in the protein content and accelerated amino acid release.

Considering these clinical advantages of LATG, we hypothesized that LATG might minimize the changes in the 
body composition after surgery. To confirm our hypothesis, the effects of LATG on body composition were investigated and compared to those of OTG. To minimize variability in perioperative care, all patients received the same fast-track surgery program in this study.

\section{Patients and Methods}

Patients. The patients were selected from the prospective database of the Kanagawa Cancer Center, Department of Gastrointestinal Surgery, Yokohama, Japan, according to the following criteria: (1) histologically-proven gastric adenocarcinoma according to the 14th edition of the general rules for gastric cancer published by the Japanese Gastric Cancer Association (11); (2) a history of total gastrectomy with curative lymph node dissection for gastric cancer as a primary treatment between May 2011 and September 2016, and (3) data available for a body composition analysis performed within one week before surgery and at one week, one month, and three month after surgery.

Surgical procedure. The patients underwent OTG or LATG with Roux-en-Y reconstruction. The extent of dissection principally followed the third edition of the Gastric Cancer Treatment Guidelines published by the Japanese Gastric Cancer Association (12). D1 or greater nodal dissection was applied for clinical stage IA tumors, and D2 dissection was applied for clinical stage IB tumors, regardless of the approach. Spleen-preserving D2 total gastrectomy was permitted in this study.

For laparoscopic surgery, one of two certified laparoscopic staff surgeons were responsible for the surgical quality. Five or six ports were used. Lymph node dissection was performed in the laparoscopic field. The omentum was preserved except where resection was necessary for lymph node dissection along the right gastroepiploic artery. A small abdominal incision $(\leq 6 \mathrm{~cm})$ was made in the upper abdomen to remove the specimen and perform reconstruction (13). For the open surgical procedure, an upper abdominal median incision extending from the xiphoid to the navel was created. The nodal dissection and reconstruction procedure were the same as that used in the laparoscopic approach. For both procedures, in principal, no drain was used. If the surgeon needed to place a drain in order to manage postoperative bleeding or pancreatitis, a low-vacuum drainage system was left in the subhepatic area for peritoneal fluid collection.

Perioperative care. The patients received the same enhanced recovery after surgery (ERAS) protocol after both OTG and LATG. The details of this protocol have been previously reported (14). In brief, the patients were allowed to eat until midnight on the day before surgery and then were required to drink the contents of two $500-\mathrm{ml}$ plastic bottles containing oral rehydration solution by $3 \mathrm{~h}$ before surgery. The nasogastric tube was removed immediately after surgery. Oral intake was initiated on postoperative day (POD) 2, beginning with water and an oral nutritional supplement. The patients began to eat solid food on POD 3, starting with rice gruel and soft food on POD 3 and advancing in three steps to regular food intake on POD 7. The patients were discharged when they had achieved adequate pain relief and soft food intake, had returned to their preoperative mobility level, and exhibited normal laboratory data on POD 7.
The evaluation of the operative morbidity and mortality. The surgical and nonsurgical complications were assessed prospectively and classified according to the Clavien-Dindo classification (15). Operative mortality was defined as postoperative death from any cause within 30 days after surgery or during the same hospital stay.

Body composition analyses. The segmental body composition was analyzed using an MC-190EM bioelectrical impedance analyzer (Tanita, Tokyo, Japan), which provides relative information regarding the amount of lean and fat tissue in the trunk area and each limb, as well as the overall body composition and hydration status. The body weight and composition were evaluated by the bioelectrical impedance analyzer within one week before surgery, at one week after surgery, at one month after surgery, and at three months after surgery.

Evaluations, statistical analyses, and ethics. Body weight (BW) loss was defined as follows: $\% \mathrm{BW}$ loss $=$ (preoperative $\mathrm{BW}-\mathrm{BW}$ at 1 week, 1 month, and 3 months after surgery) $\times 100$ /preoperative body weight. Lean body mass (LBM) loss was defined as follows: \% LBM loss=(preoperative LBM - LBM at 1 week, 1 month, and 3 months after surgery) $\times 100$ /preoperative $\mathrm{LBM}$. The values are expressed as the median and the range. The statistical analyses were performed using the chi-square test or the Wilcoxon signed-rank test. A $p$-value of less than 0.05 was considered to indicate statistical significance. The SPSS software package (version 12.0 J Win; SPSS, Chicago, IL, USA) was used for all statistical analyses. The R category and extent of dissection were determined by the Japanese Classification of Gastric Carcinoma, third English edition and the Japanese Gastric Cancer Association guidelines. The study was approved by the Institutional Review Board of Kanagawa Cancer Center.

\section{Results}

Background characteristics. A total of 303 patients were examined in this study. Two hundred and eight received OTG, and 95 received LATG. The background characteristics and baseline data are shown in Table I. Although the clinical T factor and $\mathrm{N}$ factor were significantly different, the other clinical factors were similar between the two groups. No patients had any history of weight loss, appetite loss, or food intake loss before surgery.

Surgical and pathological outcomes. Three patients assigned to receive LATG underwent conversion to open surgery. The median duration of surgery was significantly longer in the LATG group than in the OTG group $(p<0.001)$. In contrast, the median amount of bleeding was significantly less in the LATG group than in the OTG group $(p=0.002)$ (Table II). The pathological $\mathrm{T}$ factor and pathological $\mathrm{N}$ factor were also different between the two groups.

No mortalities occurred in either group. The surgical morbidities are shown in Table III. The incidences of surgical complications were similar between the two groups.

Body composition changes. The median \% BW loss (range) was $-4.7 \%(-31.5$ to 3.2$)$ at 1 week after surgery, $-8.0 \%$ (18.5 to 5.3$)$ at 1 month after surgery, and $-11.9 \%$ ( -26.6 to 
Table I. A comparison of the patients' backgrounds.

\begin{tabular}{|c|c|c|c|c|}
\hline & All cases $(n=303,(\%))$ & LATG $(\mathrm{n}=95,(\%))$ & OTG $(\mathrm{n}=208,(\%))$ & $p$-Value \\
\hline Age, years (median, range) & $69(31-86)$ & $69(45-84)$ & $70(31-86)$ & 0.630 \\
\hline Gender & & & & 0.654 \\
\hline Male & $222(73.2)$ & $68(71.6)$ & $154(74.0)$ & \\
\hline Female & $81(26.8)$ & $27(28.4)$ & $54(26.0)$ & \\
\hline ASA-PS & & & & 0.542 \\
\hline 1 & $61(20.1)$ & $21(22.1)$ & $40(19.2)$ & \\
\hline 2 & $235(77.6)$ & $73(76.8)$ & $162(77.9)$ & \\
\hline 3 & $7(2.3)$ & $1(1.1)$ & $6(2.9)$ & \\
\hline Total body weight, kg (median, range) & $58.5(31.8-89.9)$ & $59.5(36.8-89.9)$ & $58(31.8-84.9)$ & 0.200 \\
\hline Lean body mass, $\mathrm{kg}$ (median, range) & $46.5(27.7-66.8)$ & $46.9(28.2-66.8)$ & $46.0(27.7-60)$ & 0.206 \\
\hline Clinical $\mathrm{T}$ factor & & & & $<0.001$ \\
\hline $\mathrm{T} 1$ & $143(47.2)$ & $92(96.8)$ & $51(24.5)$ & \\
\hline $\mathrm{T} 2$ & $160(52.8)$ & $3(3.2)$ & $157(75.5)$ & \\
\hline Clinical $\mathrm{N}$ factor & & & & $<0.001$ \\
\hline Negative & $208(68.6)$ & $93(97.9)$ & $115(55.3)$ & \\
\hline Positive & $95(31.4)$ & $2(2.1)$ & $93(44.7)$ & \\
\hline \multicolumn{5}{|l|}{ Co-morbidity } \\
\hline Diabetes mellitus & $39(12.9)$ & $8(8.4)$ & $31(14.9)$ & 0.270 \\
\hline COPD & $69(22.8)$ & $16(16.8)$ & $53(25.5)$ & 0.529 \\
\hline Smoking habit & & & & 0.531 \\
\hline Yes & $155(51.2)$ & $51(53.7)$ & $104(50.0)$ & \\
\hline No & $148(48.8)$ & $44(46.3)$ & $104(50.0)$ & \\
\hline
\end{tabular}

ASA-PS: ASA physical status; COPD: chronic obstructive pulmonary disease; LATG: laparoscopy-assisted total gastrectomy; OTG: open total gastrectomy.

Table II. Surgical and pathological outcomes.

\begin{tabular}{|c|c|c|c|c|}
\hline & All cases $(n=303,(\%))$ & LATG $(\mathrm{n}=95,(\%))$ & OTG $(\mathrm{n}=208,(\%))$ & $p$-Value \\
\hline Lymph node dissection & & & & $<0.001$ \\
\hline $\mathrm{D} 1+$ dissection & $174(57.4)$ & $95(100)$ & $79(38.0)$ & \\
\hline $\mathrm{D} 2$ dissection & $129(42.6)$ & $0(0)$ & $129(62.0)$ & \\
\hline Bleeding, g (median) (range) & $260(5-3780)$ & $77.5(5-800)$ & $385(30-3780)$ & $<0.001$ \\
\hline Operation time, min (median) (range) & $198(80-585)$ & $234(122-394)$ & $182(80-585)$ & $<0.001$ \\
\hline Number of harvested lymph nodes (median, range) & $49(2-184)$ & $44(7-115)$ & $49(2-184)$ & \\
\hline Pathological $\mathrm{T}$ factor & & & & $<0.001$ \\
\hline $\mathrm{T} 1$ & $134(44.2)$ & $83(87.4)$ & $51(24.5)$ & \\
\hline $\mathrm{T} 2$ & $169(55.8)$ & $12(12.6)$ & $157(75.5)$ & \\
\hline Pathological N factor & & & & $<0.001$ \\
\hline Negative & $186(61.4)$ & $84(88.4)$ & $102(49.0)$ & \\
\hline Positive & $117(38.6)$ & $11(11.6)$ & $106(51.0)$ & \\
\hline
\end{tabular}

LATG: Laparoscopy-assisted total gastrectomy; OTG: open total gastrectomy.

5.8) at 3 months after surgery in the LATG group, while the median \% BW loss (range) was $-4.7 \%(-9.7$ to 1.8$)$ at 1 week after surgery, $-8.2 \%(-14.9$ to -2.4$)$ at 1 month after surgery, and $-11.6 \%(-22.7$ to 0.9$)$ at 3 months after surgery in the OTG group (Figure 1). When comparing the \% BW loss between the 2 groups, no significant differences were observed at any point $(p=0.698, p=0.528$, and $p=0.534$ at 1 week, 1 month, and 3 months, respectively). Furthermore, the median \% LBM loss (range) was $-4.2 \%(-20.0 \%$ to $17.2 \%)$ at 1 week after surgery, $-6.4 \%(-18.6 \%$ to $12.7 \%)$ at 1 month after surgery, and $-7.4 \%(-21.8 \%$ to $20.0 \%)$ at 3 months after surgery in the LATG group, while the median $\%$ BW loss (range) was $-4.0 \%(-8.6 \%$ to $9.9 \%)$ at 1 week after surgery, $-5.8 \%(-15.7 \%$ to $2.7 \%)$ at 1 month after surgery, and $-6.2 \%(-17.2 \%$ to $8.4 \%)$ at 3 month after surgery in the LATDG group (Figure 2). When comparing the \% 


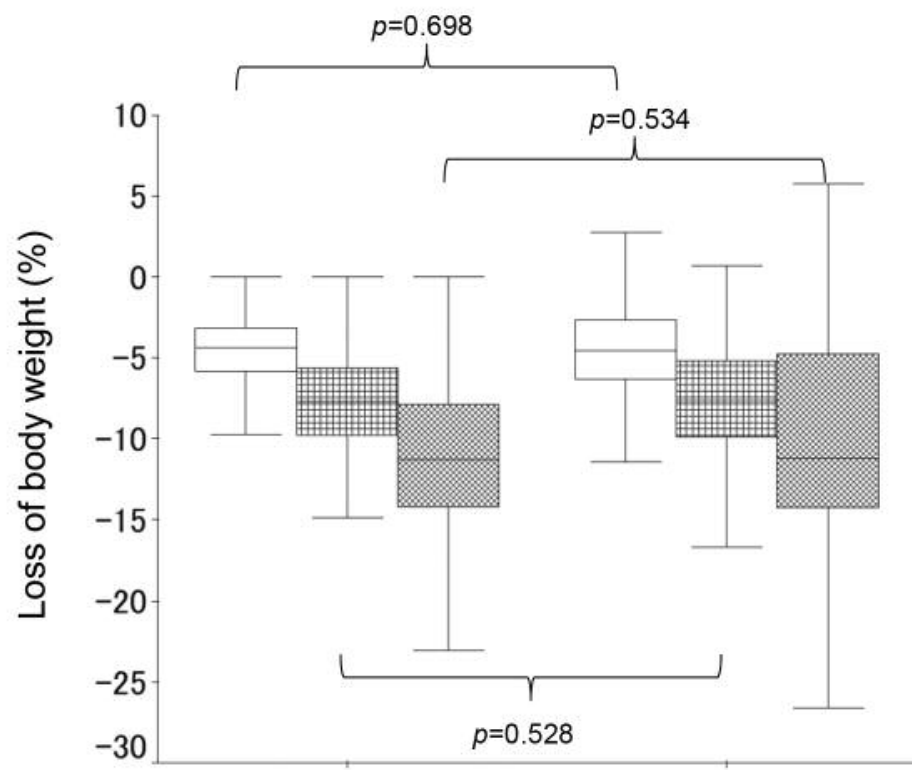

Conventional total gastrectomy Laparoscopic assisted total gastrectomy

\begin{tabular}{|c|c|c|c|}
\hline & Conventional total gastrectomy & $\begin{array}{c}\text { Laparoscopic assisted total } \\
\text { gastrectomy }\end{array}$ & -Value \\
\hline 1 week & $-4.7 \%(-31.5$ to 3.2$)$ & $-4.7 \%(-9.7$ to 1.8$)$ & 0.698 \\
\hline 1 month & $-8.0 \%(-18.5$ to 5.3$)$ & $-8.2 \%(-14.9$ to -2.4$)$ & 0.528 \\
\hline 3 months & $-11.9 \%(-26.6$ to 5.8$)$ & $-11.6 \%(-22.7$ to 0.9$)$ & 0.534 \\
\hline
\end{tabular}

Figure 1. A comparison of the changes in \% body weight loss between the laparoscopy-assisted total gastrectomy group and the open total gastrectomy group.

LBM loss between the 2 groups, no significant differences were observed at any point $(p=0.503, p=0.588$, and $p=0.946$ at 1 week, 1 month, and 3 months, respectively).

\section{Discussion}

This is the first study to compare the body composition changes after LATG and OTG in patients receiving the same perioperative care of fast-track surgery. In the present study, the body composition changes after surgery were not significantly different between the two groups, suggesting that the body composition outcomes were similar between OTG and LATG using the same perioperative care of fasttrack surgery.

We recently reported similar results between laparoscopyassisted distal gastrectomy (LADG) and open distal gastrectomy (ODG) for gastric cancer (16). The previous study compared the loss of body weight and lean body mass in patients who received LADG and ODG in a randomized cohort at a single institution. Furthermore, all patients received the same perioperative care via a fast-track surgery protocol. In that study, the loss of body weight and lean body
Table III. A comparison of morbidity and mortality between the ODG and $L A D G$ groups.

\begin{tabular}{lccc}
\hline & $\begin{array}{c}\text { LATG } \\
(\mathrm{n}=95,(\%))\end{array}$ & $\begin{array}{c}\text { OTG } \\
(\mathrm{n}=208,(\%))\end{array}$ & $p$-Value \\
\hline Total & $20(21.1)$ & $52(25.0)$ & 0.356 \\
Pancreatic fistula & $6 / 0 / 0 / 0(6.3)$ & $11 / 2 / 0 / 0(6.3)$ & \\
Anastomotic leakage & $0 / 7 / 1 / 0(8.4)$ & $4 / 6 / 2 / 0(5.8)$ & \\
Anatomic stenosis & $0 / 2 / 0 / 0(2.1)$ & $0 / 2 / 0 / 0(1.0)$ & \\
Abdominal abscess & $2 / 0 / 0 / 0(2.1)$ & $1 / 4 / 0 / 0(2.4)$ & \\
Ileus & $0 / 3 / 0 / 0(3.2)$ & $4 / 3 / 1 / 0(3.8)$ & \\
Pneumonia & $2 / 1 / 1 / 0(4.2)$ & $5 / 1 / 0 / 0(2.9)$ & \\
Surgical site infection & $1 / 0 / 0 / 0(1.1)$ & $2 / 0 / 0 / 0(1.0)$ & \\
Postoperative bleeding & $0 / 0 / 0 / 0(0)$ & $4 / 1 / 0 / 0(2.4)$ & \\
Others & $0 / 2 / 0 / 0(2.1)$ & $6 / 0 / 0 / 0(2.9)$ & \\
\hline
\end{tabular}

LATG: Laparoscopy-assisted total gastrectomy; OTG: open total gastrectomy. *There is some overlapping in the morbidity.

mass in the LADG and ODG groups did not differ significantly. Although the present study was not a randomized prospective study, our results from these two 


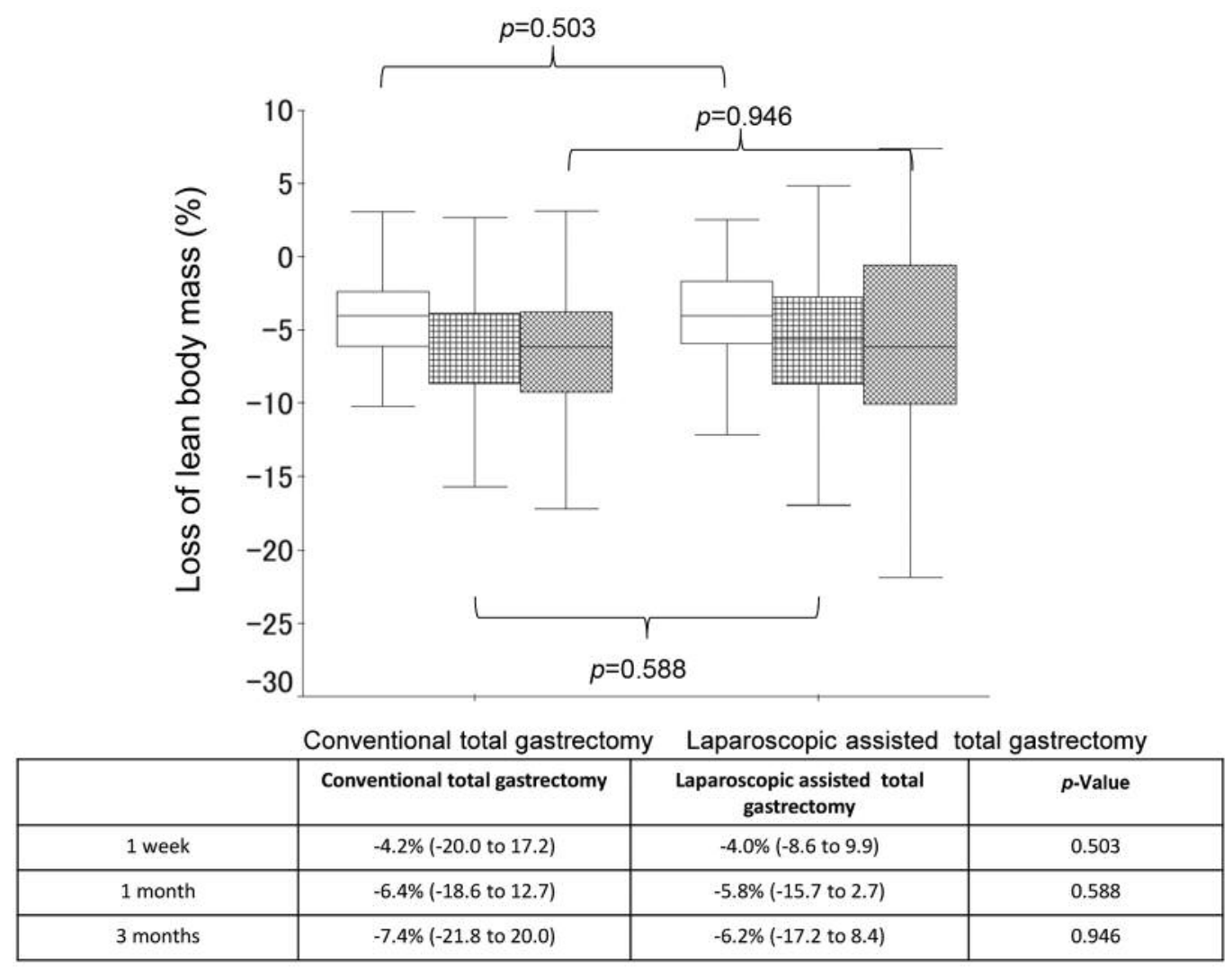

Figure 2. A comparison of the changes in \% lean body mass loss between the laparoscopy-assisted total gastrectomy group and the open total gastrectomy group.

studies strongly suggest that the loss of body weight and lean body mass observed with an open approach are not attenuated by a laparoscopic approach.

There are several possible reasons for the similarities in body composition changes between the LATG and OTG groups. First, the surgical stress might actually be similar between the two approaches. Some randomized studies comparing laparoscopic and open surgery have reported that there were no differences in the systemic immune response between the two approaches (17). Whether or not laparoscopic surgery is associated with less surgical stress than open surgery remains controversial. Second, differences in the operative time may have contributed to our findings. In the present study, the operative time was significantly longer in the LATG group than in the OTG group, which may have reduced the positive effects of the less-invasive nature of LATG. Third, the creation of a wound incision contributed to a smaller induced surgical stress response. Both surgical approaches involve similar procedures for performing visceral dissection, mobilization, and anastomosis, with the exception of the length of the skin incision. Body composition changes might, therefore, not be affected by the skin incision alone.
Postoperative complications and perioperative details are strongly related to body composition changes, especially loss of lean body mass after surgery. When comparing the incidence and details of postoperative surgical complications between the present study and a retrospective cohort study based on a nationwide registry database in Japan, similar results were observed (18). The incidence of grade 2 or higher postoperative surgical complications were $25.5 \%$ in the OTG group and $21.1 \%$ in the LATG group, while the incidence of grade 2 or higher postoperative surgical complications was $16.4 \%$ to $19 \%$ in the OTG group and $17.8 \%$ to $20.1 \%$ in the LATG group, in the present study. In addition, the incidence of pneumonia $(2.8 \%-4.2 \%$ vs. $2.0 \%-3.5 \%)$, anatomic leak (5.8\%-6.3\% vs. $3.6 \%-5.7 \%)$, and abdominal abscess (2.1\%$2.4 \%$ vs. $4.0 \%-5.9 \%$ ) were similar between the present study and the previous study. Furthermore, the perioperative details between the present and previous studies, the results were also similar. Therefore, the results of the present study results might be compatible with those of a retrospective cohort study based on a nationwide registry database in Japan (18).

Several limitations associated with the present study warrant mention. First, the sample size may not have been 
large enough to lead to definitive conclusions, although the current results were obtained while using the same perioperative care of fast-track surgery. Second, the segmental body composition was analyzed using a bioelectrical impedance analyzer, which considers not only the muscle mass but also the liver and kidney masses. The mass of visceral organs is not changed by either of these surgeries, so the major contributor to any changes in the lean body mass would be muscle, and a bioelectrical impedance analyzer cannot directly measure muscle mass. Third, surgical stress and oral intake (in calories) after surgery were not measured. The exact mechanisms underlying body composition changes are unknown. Fourth, this study was a single-center study. Given these limitations, confirmation of the present results a multi-center prospective cohort study is necessary.

In conclusion, the body composition changes were similar between the OTG and LATG groups in a randomized comparison using the same perioperative care of fast-track surgery. The benefits of LATG should be clarified and discussed in the future.

\section{Conflicts of Interest}

The Authors have no conflicts of interest to disclose regarding this study.

\section{Acknowledgements}

This work was supported in part by the Non-Governmental Organizations Kanagawa Standard Anti-cancer Therapy Support System. The Authors express their sincere gratitude to Ms. Natsumi Sato and Ms. Rika Takahashi for their excellent data management in this study.

\section{References}

1 Torre LA, Bray F, Siegel RL, Ferlay J, Lortet-Tieulent J and Jemal A: Global cancer statistics, 2012. CA Cancer J Clin 65: 87-108, 2015.

2 Yoshikawa T, Rino Y, Yukawa N, Oshima T, Tsuburaya A and Masuda M: Neoadjuvant chemotherapy for gastric cancer in Japan: a standing position by comparing with adjuvant chemotherapy. Surg Today 44: 11-21, 2014.

3 Aoyama T and Yoshikawa T: Adjuvant therapy for locally advanced gastric cancer. Surg Today 47: 1295-1302, 2017.

4 Kitano S, Iso Y, Moriyama M and Sugimachi K: Laparoscopyassisted. Billroth-I gastrectomy. Surg Laparosc Endosc 4: 146$148,1994$.

5 Kodera Y, Fujiwara M, Ohashi N, Nakayama G, Koike M, Morita $\mathrm{S}$ and Nakao A: Laparoscopic surgery for gastric cancer: a collective review with meta-analysis of randomized trials. J Am Coll Surg 211: 677-686, 2010.

6 Adachi Y, Shiraishi N, Shiromizu A, Bandoh T, Aramaki M and Kitano S: Laparoscopy-assisted Billroth I gastrectomy compared with conventional open gastrectomy. Arch Surg 135: 806-810, 2000.

7 Aoyama T, Yoshikawa T, Shirai J, Hayashi T, Yamada T, Tsuchida K, Hasegawa S, Cho H, Yukawa N, Oshima T, Rino Y, Masuda M and Tsuburaya A: Body weight loss after surgery is an independent risk factor for continuation of S-1 adjuvant chemotherapy for gastric cancer. Ann Surg Oncol 20: 2000-2006, 2013.

8 Aoyama T, Kawabe T, Fujikawa H, Hayashi T, Yamada T, Tsuchida K, Yukawa N, Oshima T, Rino Y, Masuda M, Ogata T, Cho H and Yoshikawa T: Loss of lean body mass as an independent risk factor for continuation of S-1 adjuvant chemotherapy for gastric cancer. Ann Surg Oncol 22: 2560-2566, 2015.

9 Aoyama T, Sato T, Maezawa Y, Kano K, Hayashi T, Yamada T, Yukawa N, Oshima T, Rino Y, Masuda M, Ogata T, Cho H and Yoshikawa T: Postoperative weight loss leads to poor survival through poor S-1 efficacy in patients with stage II/III gastric cancer. Int J Clin Oncol 22: 476-483, 2017.

10 Castell JV, Gómez-Lechón MJ, David M, Andus T, Geiger T, Trullenque R, Fabra R and Heinrich PC: Interleukin-6 is the major regulator of acute phase protein synthesis in adult human hepatocytes. FEBS Lett 242: 237-239, 1989.

11 Japanese Gastric Cancer Association: Japanese gastric cancer treatment guidelines 2010 (ver. 3). Gastric Cancer 14: 113-123, 2011.

12 Japanese Gastric Cancer Association: Japanese classification of gastric carcinoma: 3rd English edition. Gastric Cancer 14: 101112, 2011.

13 Yoshikawa T, Hayashi T, Aoyama T, Cho H, Fujikawa H, Shirai J, Hasegawa S, Yamada T, Oshima T, Yukawa N, Rino Y, Masuda M, Ogata T and Tsuburaya A: Laparoscopic esophagojejunostomy using the EndoStitch and a circular stapler under a direct view created by the ENDOCAMELEON. Gastric Cancer 16: 609-614, 2013.

14 Yamada T, Hayashi T, Cho H, Yoshikawa T, Taniguchi H, Fukushima R and Tsuburaya A: Usefulness of enhanced recovery after surgery protocol as compared with conventional perioperative care in gastric surgery. Gastric Cancer 15: 34-41, 2012.

15 Clavien PA, Barkun J, de Oliveira ML, Vauthey JN, Dindo D, Schulick RD, de Santibañes E, Pekolj J, Slankamenac K, Bassi C, Graf R, Vonlanthen R, Padbury R, Cameron JL and Makuuchi M: The Clavien-Dindo classification of surgical complications: five-year experience. Ann Surg 250: 187-196, 2009.

16 Aoyama T, Sato T, Hayashi T, Yamada T, Cho H, Ogata T, Oba $\mathrm{K}$ and Yoshikawa $\mathrm{T}$ : Does a laparoscopic approach attenuate the body weight loss and lean body mass loss observed in open distal gastrectomy for gastric cancer? a single-institution exploratory analysis of the JCOG 0912 phase III trial. Gastric Cancer 21: 345-352, 2018.

17 Aoyama T, Yoshikawa T, Hayashi T, Hasegawa S, Tsuchida K, Yamada T, Cho H, Ogata T, Fujikawa H, Yukawa N, Oshima T, Rino $\mathrm{Y}$ and Masuda M: Randomized comparison of surgical stress and the nutritional status between laparoscopy-assisted and open distal gastrectomy for gastric cancer. Ann Surg Oncol 21: 1983-1990, 2014.

18 Kodera Y, Yoshida K, Kumamaru H, Kakeji Y, Hiki N, Etoh T, Honda M, Miyata H, Yamashita Y, Seto Y, Kitano S and Konno $\mathrm{H}$ : Introducing laparoscopic total gastrectomy for gastric cancer in general practice: a retrospective cohort study based on a nationwide registry database in Japan. Gastric Cancer, 2018. doi: 10.1007/s10120-018-0795-0. [Epub ahead of print]

Received July 17, 2018

Revised August 2, 2018

Accepted August 7, 2018 